\title{
Caracterización y análisis de revistas de investigación jurídicas en México indexadas en Scopus [2008-2018]*
}

Characterization and analysis of law research journals in Mexico

indexed in Scopus [2008-2018]

César Jiménez-Yañez ${ }^{* *}$

Resumen: Como una forma de reflexionar en torno al nuevo paradigma editorial científico, a continuación presento una caracterización y análisis de las revistas de investigación jurídica en México que se encuentran indexadas en la base de datos Scopus desde el año 2008 hasta el 2018; diez años que dan cuenta de la evolución editorial académica en este ámbito disciplinar. El objetivo es identificar elementos y características específicas de estas publicaciones, derivados de su perfil y de una sistematización, para analizar y tomar de decisiones para otras revistas que aspiran a integrar este índice.

Palabras clave: divulgación, indexación, producción científica.

Abstract: As a way of reflecting on the new scientific editorial paradigm, below, I present a characterization and analysis of the legal research journals in Mexico, that are indexed in the Scopus database from 2008 to 2018; ten years that highlight the academic editorial evolution of this disciplinary field. The objective is to identify specific elements and characteristics of these publications, derived from their profile and from a systematization, which will serve for the analysis and decision making for other journals that aspire to integrate this index.

Keywords: Dissemination, indexing, scientific production

\footnotetext{
* Artículo de investigación Número Especial de Aniversario. Recibido el 20 de mayo de 2020 y aceptado para su publicación el 17 de septiembre de 2020.

** Coordinador editorial en el Instituto de Investigaciones Culturales-Museo de la Universidad Autónoma de Baja California, México. / jimenez.cesar@uabc.edu.mx / orcid.org/0000-0002-4018-3126
} 


\section{Sumario}

1. Introducción

2. Identificación de las revistas

3. Caracterización de las revistas de investigación jurídica mexicanas indexadas en Scopus

4. Reflexiones finales

5. Referencias

\section{Introducción}

Uno de los principales criterios de calidad y visibilidad que debe tener una revista científica a nivel internacional es estar integrada en un sistema de información que sea comprensivo o especializado, y selectivo o exhaustivo; es decir, una base de datos que indexe y genere indicadores métricos confiables y válidos. Bajo esta descripción general, la comunidad científica internacional reconoce las bases de datos Scopus con su plataforma de medición Scimago Journal \& Country Rank (SJR) y Web of Science con Journal Citaton Report (JCR). Estas dos bases de datos y sus respectivos sistemas estadísticos, en los últimos diez años, han provocado - sobre todo en América Latina - una verdadera "fiebre del oro" o "revolución científica" en las áreas de ciencias sociales y humanidades. Esta nueva forma de ver y entender la visibilidad, el posicionamiento e impacto de las revistas científicas ha sido promovido en diversos países a través de la implantación y puesta en marcha de políticas públicas con la asesoría, en algunos de los casos, de Scimago Lab ${ }^{1}$ (el caso de México, Chile y Colombia), estableciéndose un nuevo discurso basado en los conceptos de impacto, calidad y visibilidad de una revista científica.

Este nuevo discurso ha permeado las bases de todo el proceso editorial y el de la construcción y producción de conocimiento, a tal punto que se considera que, si una revista dice ser de calidad y no integra una de estas bases de datos, no puede adjudicarse este calificativo y, por ende, si un autor no publica en alguna de estas revistas, su trabajo "no es de calidad" y no cuenta para los incentivos, promociones,

${ }_{1}$ De acuerdo con el portal de Universia, Scimago Lab es "una empresa de base tecnológica que ofrece soluciones innovadoras para mejorar la visibilidad científica y la reputación en línea". Consultado y disponible en: https://www.universia.es/investigacion/profesores/at/1150940. 
becas o estímulos relacionados con la producción personal del docente 0 investigador. Esta situación ha generado en el mundo varios movimientos de reflexión crítica en torno a este tema (DORA, ${ }^{2} \mathrm{AMELICA}^{3}$ y Plan $\mathrm{S}^{4}$ ). A pesar de ello, esta maquinaria sigue avanzando, supeditando los procesos administrativos, académicos y editoriales de las instituciones a una forma globalizada (capitalista/mercantil) de concebir y divulgar la ciencia. En el caso de México así ha sido, y hoy la política pública restringe y supedita sus recursos a esta forma de entender la producción científica y su calidad, lo que ha llevado a las revistas de investigación y a los investigadores a reorientar sus esfuerzos académicos y administrativos para alcanzar este nirvana que se representa y simboliza en Scopus y Web of Science. Para Nassi-Caló, ${ }^{5}$ esto también es culpa de los investigadores, porque son ellos quienes contribuyen a este círculo vicioso: "cuando se les solicita identificar sus publicaciones más relevantes, ellos generalmente las seleccionan en base a los índices de citaciones, en lugar de atribuir a sus artículos su verdadera importancia académica o un descubrimiento particularmente innovador".

No debemos olvidar que más allá de los objetivos específicos que persigue una revista académica y de los requerimientos internacionales, las revistas, aparte de comunicar, divulgar, diseminar y difundir, son el espacio de legitimación y almacenamiento del conocimiento científico, y permiten el avance de la ciencia y de los científicos, abriendo espacios de oportunidad para seguir creando y desarrollando la ciencia. ${ }^{6}$ Todo lo anterior queda en tela de juicio cuando se ha implementado el mismo discurso, sistema de evaluación y medición, desde las ciencias naturales y exactas (para quienes este discurso encuentra mayor sentido), para las ciencias sociales y humanidades, donde las especificidades de hacer ciencia y generar conocimiento son distintas.

\footnotetext{
2 Para más información, revisar: https://sfdora.org/.

${ }^{3}$ Para más información, revisar: http://amelica.org/.

${ }^{4}$ Para más información, revisar: https://www.coalition-s.org/.

${ }^{5}$ Nassi-Calò, Lilian, "La miopía de los indicadores bibliométricos" [online], SciELO en Perspectiva, 2017. Disponible en: https://blog.scielo.org/es/2017/06/01/la-miopia-de-los-indicadoresbibliometricos/.

${ }^{6}$ López-Ornelas, Maricela y Cordero-Arroyo, Graciela, "Un intento por definir las características generales de las revistas académicas electrónicas", Revista Razón y Palabra, 2005, vol. 10, núm. 43, pp. 1-33. Disponible en: http://eprints.rclis.org/15700/.
} 
Como una forma de reflexionar en torno a este nuevo paradigma editorial científico, a continuación presento una caracterización y análisis de las revistas de investigación jurídicas en México que se encuentran indexadas en la base de datos Scopus desde el año 2008 hasta el 2018, diez años que dan cuenta de la evolución editorial académica de este ámbito disciplinar. El objetivo es entregar elementos objetivos, derivados de una sistematización y caracterización de estas revistas, que sirvan para el análisis y toma de decisiones para otras revistas que aspiran a integrar este índice.

Lo anterior, considerando que el Sistema de Clasificación de Revistas Mexicanas de Ciencia y Tecnología, del Conacyt, ${ }^{7}$ de acuerdo con su clasificación temática, reconoce 16 revistas que se relacionan con el ámbito del derecho, y solo seis de estas se encuentran indexadas en Scopus, es decir, 35\% del total. Esto nos lleva a cuestionarnos cuáles son los elementos distintivos que tienen estas revistas por sobre las otras, considerando que para integrar la base de datos Scopus se debe solicitar una pre-evaluación.

\section{Identificación de las revistas}

Si bien el contenido y los temas abordados marcan las tendencias de una disciplina, también es necesario considerar las características estructurales que poseen las revistas científicas que son parte de plataformas de evaluación (bases de datos o repositorios) y que son aceptadas como "las mejores". Al reconocerlas, podemos identificar algunos elementos objetivos que nos permiten retratarlas para que otras revistas que aspiran a integrar estos índices puedan conocerlas. Actualmente aparecen, ${ }^{8}$ de acuerdo con la información en la base de datos Scimago Journal \& Country Rank ${ }^{9}$ (ver tabla 1 ), seis revistas asociadas al área de ciencias sociales y a la categoría "jurídica/legal” o del "derecho" en México, indexadas en Scopus.

Tabla 1. Revistas de investigación jurídica mexicanas indexadas en Scopus.

\footnotetext{
${ }^{7}$ Para más información revisar el Sistema de Clasificación de Revistas Mexicanas de Ciencia y Tecnología del Conacyt. Ver http://www.revistascytconacyt.mx/

${ }^{8}$ Sistematización de datos, revisión y consulta de base de datos hecha en mayo de 2020.

${ }^{9}$ Para más información revisar https://www.scimagojr.com/
} 


\section{Nombre}

Boletín Mexicano de Derecho Comparado

Cuestiones Constitucionales

Anuario Mexicano de Derecho Internacional Norteamérica

Problema

Revista Latinoamericana de Derecho Social

ISSN impreso ISSN digital institución

\begin{tabular}{|l|l|l|}
\hline $0041-8633$ & $2448-4873$ & UNAM / IIJ \\
\hline $1405-9193$ & $2448-4881$ & UNAM / IIJ \\
\hline $1870-4654$ & $2448-7872$ & UNAM / IIJ \\
\hline $1870-3550$ & $2448-7228$ & UNAM / CISAN \\
\hline $2007-4387$ & $2448-7937$ & UNAM / IIJ \\
\hline $1870-4670$ & $2448-7899$ & UNAM / IIJ \\
\hline
\end{tabular}

Fuente: Elaboración propia con base en datos de Scimago Journal \& Country Rank.

Uno de los datos llamativos es que las seis revistas que aparecen en Scimago son trabajadas editorialmente dentro de la Universidad Nacional Autónoma de México (UNAM); cinco, al alero del Instituto de Investigaciones Jurídicas (IIJ) y una en el Centro de Investigaciones sobre América del Norte (CISAN). La primera revista en ingresar a Scopus fue el Boletín Mexicano de Derecho Comparado, y lo hizo en 2009; se mantiene hasta 2018, con siete años en el Quartil 4 (2010 al 2013 y del 2015 al 2017) y dos años en el Quartil 3 (2014 y 2018). A continuación (tabla 2), presento la relación de ingreso y clasificación actual de las seis revistas en Scopus.

Tabla 2. Año de ingreso y quartil de las revistas de investigación jurídica mexicanas indexadas en Scopus.

Nombre revista

\begin{tabular}{|c|c|c|c|c|c|c|c|c|c|c|c|}
\hline \multirow[t]{2}{*}{ Nombre revista } & \multicolumn{11}{|c|}{ AÑNOS } \\
\hline & 2008 & 2009 & 2010 & 2011 & 2012 & 2013 & 2014 & 2015 & 2016 & 2017 & 2018 \\
\hline Boletín Mexicano de Derecho Comparado & & I & Q4 & Q4 & Q4 & $\mathrm{Q} 4$ & Q3 & Q4 & Q4 & Q4 & Q3 \\
\hline Cuestiones Constitucionales & & & & & & $\mathbf{I}$ & Q4 & Q4 & Q4 & Q4 & Q4 \\
\hline Anuario Mexicano de Derecho Internacional & & & & & & & $\mathbf{I}$ & $\mathrm{Q} 4$ & $\mathrm{Q} 4$ & Q3 & Q3 \\
\hline Norteamerica & & & & & & & & & $\mathbf{I}$ & $\mathrm{SC}$ & Q4 \\
\hline Problema & & & & & & & & & $\mathbf{I}$ & Q4 & Q4 \\
\hline Revista Latinoamericana de Derecho Social & & & & & & & & & I & Q4 & $\mathrm{Q} 4$ \\
\hline
\end{tabular}

I: Ingreso / SC: Sin Clasificación / Q4: Quartil 4 / Q3: Quartil 3

Fuente: Elaboración propia con base en datos de la plataforma Scimago Journal \& Country Rank.

De acuerdo con esta información, la revista que mejor ha avanzado en la clasificación es el Anuario Mexicano de Derecho Internacional, que después de tres 
años desde su ingreso pasó a estar en Q3, manteniéndose por dos años consecutivos. Es importante destacar y recordar que la fórmula de cálculo para las citas en Scopus considera una ventana de tres años, lo que significa -o por lo menos podríamos interpretar-que es normal que una revista que inicia en el cuartil 4 y que cumple con los criterios en cada evaluación anual demore tres o más años en obtener citas y pasar al siguiente cuartil. Considerando esto último, es importante revisar la cantidad de citas por años que han obtenido desde que ingresaron a la base de datos (ver tabla 3), como una forma de visualizar y medir su importancia e impacto en el área y categoría que representa; es decir, considerando que las únicas citas reconocidas por Scopus deben ser citas en revistas que pertenecen a la misma base de datos, podremos interpretar si su inclusión en esta base de datos le ha dado más visibilidad e injerencia en su ámbito disciplinar.

Tabla 3. Cantidad de citas por año de las revistas de investigación jurídicas mexicanas indexadas en Scopus de acuerdo con CiteScore.

Nombre revista

Boletín Mexicano de Derecho Comparado
Cuestiones Constitucionales
Anuario Mexicano de Derecho Internacional
Norteamerica
Problema
Revista Latinoamericana de Derecho Social

AÑos

$\begin{array}{lllllllllll}2008 & 2009 & 2010 & 2011 & 2012 & 2013 & 2014 & 2015 & 2016 & 2017 & 2018\end{array}$

\begin{tabular}{|c|c|c|c|c|c|c|c|c|c|c|}
\hline & & S/I & S/I & 2 & 5 & 5 & 2 & 0 & 9 & 10 \\
\hline & & & & & & 0 & 0 & 2 & 2 & 2 \\
\hline & & & & & & & 1 & 2 & 10 & 18 \\
\hline & & & & & & & & & S/I & 4 \\
\hline & & & & & & & & & 1 & 0 \\
\hline & & & & & & & & & 4 & 4 \\
\hline
\end{tabular}

S/l: Sin Información

Fuente: Elaboración propia con base en datos de la plataforma Sources Scopus Preview/CiteScore.

Como podemos apreciar, la cantidad de citas por año no es significativa en estas revistas, esto quizás se deba al poco tiempo que tienen en la base de datos. Solo la revista Anuario Mexicano de Derecho Internacional muestra un crecimiento exponencial en cuanto a citas; será necesario revisar entonces, como medida de calidad, si la cantidad de citas de cada año tiene relación con la cantidad de artículos o documentos publicados por estas revistas y que han sido considerados por Scopus (ver tabla 4), de acuerdo con su ventana de tres años. Esto, porque 
supuestamente una cita habla de la calidad del trabajo citado. Habrá que preguntarse y reflexionar si más significa mejor. La siguiente tabla muestra la cantidad de documentos considerados por Scopus en cada ventana de tiempo para las citas de un año específico. De acuerdo con la definición que hace el portal de Elsevier, de CiteScore, "se calculan las citas de todos los documentos en el año uno a todos los documentos publicados en los tres años anteriores para un título [...] para calcular un valor de 2015, CiteScore cuenta las citas recibidas en 2015 en documentos publicados en 2012, 2013 y 2014". ${ }^{10}$

Tabla 4. Cantidad de documentos publicados y considerados para las citas anuales de las revistas de investigación jurídica mexicanas indexadas en Scopus de acuerdo con CiteScore.

\begin{tabular}{|c|c|c|c|c|c|c|c|}
\hline \multirow{3}{*}{ Nombre revista } & \multicolumn{7}{|c|}{ PERIODO / RANGO } \\
\hline & 2018 & 2017 & 2016 & 2015 & 2014 & 2013 & 2012 \\
\hline & 2017-2015 & 2016-2014 & 2015-2013 & 2014-2012 & 2013-2011 & 2012-2010 & 2011-2009 \\
\hline \multirow{4}{*}{$\begin{array}{l}\text { Boletín Mexicano de Derecho Comparado } \\
\text { Cuestiones Constitucionales } \\
\text { Anuario Mexicano de Derecho Internacional } \\
\text { Norteamerica }\end{array}$} & 106 & 125 & 126 & 123 & 116 & 121 & 125 \\
\hline & 69 & 60 & 69 & 49 & 26 & $\mathbf{0}$ & $\mathbf{0}$ \\
\hline & 74 & 71 & 48 & 22 & $\mathbf{0}$ & $\mathbf{0}$ & $\mathbf{0}$ \\
\hline & 35 & $\mathbf{0}$ & & & & & \\
\hline \multirow{2}{*}{$\begin{array}{l}\text { Problema } \\
\text { Revista Latinoamericana de Derecho Social }\end{array}$} & 44 & 10 & $\mathbf{0}$ & & & & \\
\hline & 37 & 19 & & & & & \\
\hline
\end{tabular}

Fuente: Elaboración propia con base en datos de la plataforma Sources Scopus Preview/CiteScore.

De acuerdo con los datos, podemos observar que el Boletín Mexicano de Derecho Comparado, a pesar de publicar más de cien documentos por cada periodo de medición de citas, no tiene citas significativas en relación con la cantidad de documentos; a diferencia del Anuario Mexicano de Derecho Internacional, que, publicando menos documentos, tiene mayor cantidad de citas. Sin embargo, es la Revista Latinoamericana de Derecho Social la que, publicando mucho menos de la mitad que las revistas mencionadas, mantiene una cantidad de citas alta y constante; esto podría interpretarse como un rasgo de calidad de sus documentos. Esto nos lleva a plantearnos, en este análisis específico, que no importa la cantidad

\footnotetext{
10 Información disponible en https://www.elsevier.com/editors-update/story/journalmetrics/citescore-a-new-metric-to-help-you-choose-the-right-journal.
} 
de años de funcionamiento de una revista ni la cantidad de artículos que publica, sino la calidad de cada uno de sus papers, la relevancia de su contenido en la disciplina.

\section{Caracterización de las revistas de investigación jurídicas mexicanas indexadas en Scopus}

Hecho el primer análisis, es necesario, como parte de la caracterización y sistematización de los datos más importantes de cada revista, conocer los datos estructurales y de funcionamiento más significativos de estas revistas. A continuación, se presenta una breve ficha:

Ficha 1. Boletín Mexicano de Derecho Comparado

Casa editora: Instituto de Investigaciones Jurídicas-UNAM

Año de inicio: 1968

Año de ingreso a Scopus: 2009

Formato: digital / https://revistas.juridicas.unam.mx/index.php/derecho-comparado

Periodicidad: cuatrimestral

Evaluación por pares: doble ciego

Acceso: Open Access

Licencia Creative Commons: Atribución-NoComercial 4.0 Internacional

Idiomas de publicación: español, inglés, francés, italiano y portugués

Secciones: artículos, estudios legislativos, bibliografía e información

Integra las siguientes bases de datos, repositorios y catálogos de acuerdo con MIAR: ${ }^{11}$ Scopus, Dialnet, Latindex y Proquest. Además, se encuentra admitida en la base de datos SciELo México, en el Sistema de Clasificación de Revistas Mexicanas de Ciencia y Tecnología del Conacyt, con la clasificación Q3 y en la Red Iberoamericana de Innovación y Conocimiento Científico (Redib).

Ficha 2. Cuestiones Constitucionales. Revista Mexicana de Derecho Constitucional

\footnotetext{
11 Matriz de Información para el Análisis de Revistas, reúne información clave para la identificación y el análisis de revistas, y es administrada por la Facultad de Información y Medios Audiovisuales, de la Universidad de Barcelona. Se puede consultar en: http://miar.ub.edu/
} 
Casa editora: Instituto de Investigaciones Jurídicas-UNAM

Año de inicio: 1999

Año de ingreso a Scopus: 2013

Formato: digital / https://revistas.juridicas.unam.mx/index.php/cuestiones-

\section{constitucionales}

Periodicidad: semestral

Evaluación por pares: doble ciego

Acceso: Open Access

Licencia Creative Commons: Atribución-NoComercial 4.0 Internacional

Idiomas de publicación: español, inglés, francés, italiano y portugués

Secciones: artículos doctrinales, comentarios jurisprudenciales, comentarios legislativos y reseñas bibliográficas e información.

Integra las siguientes bases de datos, repositorios y catálogos de acuerdo con MIAR: Scopus, Dialnet y Latindex. Además, se encuentra admitida en la base de datos SciELo México, en el Sistema de Clasificación de Revistas Mexicanas de Ciencia y Tecnología del Conacyt, con la clasificación Q4 y en la Red Iberoamericana de Innovación y Conocimiento Científico (Redib).

Ficha 3. Anuario Mexicano de Derecho Internacional

Casa editora: Instituto de Investigaciones Jurídicas-UNAM

Año de inicio: 2000

Año de ingreso a Scopus: 2014

Formato: digital / https://revistas.juridicas.unam.mx/index.php/derecho-internacional

Periodicidad: Anual

Evaluación por pares: doble ciego

Acceso: Open Access

Licencia Creative Commons: Atribución-NoComercial 4.0 Internacional

Idiomas de publicación: español, inglés, portugués, francés e italiano

Secciones: doctrina (artículos académicos), comentarios, reseñas, práctica internacional mexicana, jurisprudencia, coyuntura internacional y polémica. 
Integra las siguientes bases de datos, repositorios y catálogos de acuerdo con MIAR: Scopus, Emerging Sources Citation Index (ESCI/WoS), Dialnet y Latindex. Además, se encuentra admitida en la base de datos SciELo México y en el Sistema de Clasificación de Revistas Mexicanas de Ciencia y Tecnología del Conacyt, con la clasificación Q3.

Ficha 4. Norteamérica. Revista Académica del CISAN-UNAM

Casa editora: Centro de Investigaciones sobre América del Norte-UNAM Año de inicio: 2006

Año de ingreso a Scopus: 2016

Formato: digital / http://www.revistanorteamerica.unam.mx/index.php/nam

Periodicidad: Semestral

Evaluación por pares: doble ciego

Acceso: Open Access

Licencia Creative Commons: Atribución-NoComercial 4.0 Internacional Idiomas de publicación: español, inglés, francés

Secciones: ensayos, análisis de actualidad, dossier, apuntes bibliográficos, notas críticas y reflexiones.

Integra las siguientes bases de datos, repositorios y catálogos de acuerdo con MIAR: Scopus, Fuente Académica Premier (EBSCO) y Latindex. Además, se encuentra admitida en el Sistema de Clasificación de Revistas Mexicanas de Ciencia y Tecnología del Conacyt, con la clasificación Q3. Norteamérica se encuentra desactualizada en las bases de datos: Dialnet (2018), Redalyc (2018), DOAJ (2017) y tiene su "indización interrumpida" en SciELo México (desde septiembre de 2019).

Ficha 5: Problema. Anuario de Filosofía y Teoría del Derecho

Casa editora: Instituto de Investigaciones Jurídicas-UNAM

Año de inicio: 2007

Año de ingreso a Scopus: 2016

Formato: digital / https://revistas.juridicas.unam.mx/index.php/filosofia-derecho

Periodicidad: Anual 
Evaluación por pares: doble ciego

Acceso: Open Access

Licencia Creative Commons: Atribución-NoComercial 4.0 Internacional

Idiomas de publicación: español, inglés.

Secciones: discusión, artículos, comentarios, entrevistas, reseñas.

Integra las siguientes bases de datos, repositorios y catálogos de acuerdo con MIAR: Esta revista no presenta datos en MIAR. Se encuentra indizada en Scopus. Además, se encuentra admitida en la base de datos SciELo México, en Latindex y en el Sistema de Clasificación de Revistas Mexicanas de Ciencia y Tecnología del Conacyt, con la clasificación Q4.

Ficha 6. Revista Latinoamericana de Derecho Social

Casa editora: Instituto de Investigaciones Jurídicas-UNAM

Año de inicio: 2005

Año de ingreso a Scopus: 2016

Formato: digital / https://revistas.juridicas.unam.mx/index.php/derecho-social

Periodicidad: Semestral

Evaluación por pares: doble ciego

Acceso: Open Access

Licencia Creative Commons: Atribución-NoComercial 4.0 Internacional

Idiomas de publicación: español, inglés.

Secciones: artículos, comentarios, reseñas bibliográficas.

Integra las siguientes bases de datos, repositorios y catálogos de acuerdo con MIAR: Scopus, Emerging Sources Citation Index (ESCI/WoS) y Dialnet. Además, se encuentra admitida en la base de datos SciELo México, en Latindex, en Redalyc, en el Sistema de Clasificación de Revistas Mexicanas de Ciencia y Tecnología del Conacyt, con la clasificación Q4, y en la Red Iberoamericana de Innovación y Conocimiento Científico (Redib). También aparece como "publicación discontinuadaen Elsevier" (2017). 
Podemos observar en las fichas que las diferencias entre estas revistas son mínimas y que la base administrativa, estructural/operativa es prácticamente la misma. La única diferencia de fondo serían las secciones y las bases de datos a las cuales están adscritas. Esto invita a la reflexión en cuanto a esta información, que no difiere tanto de la realidad de muchas otras revistas de investigación jurídicas en México que cumplen con los mismos criterios. Como el análisis no se centra en la calidad del contenido, no podemos atribuir esta característica como la principal para la inclusión de estas seis revistas en la base de datos Scopus, en detrimento de otras que cuentan también con un muy buen nivel en sus publicaciones. Esta radiografía nos entrega algunos elementos a tener en cuenta para la toma de decisiones de otras revistas que aspiran a ingresar a esta base de datos.

\section{Reflexiones finales}

Es interesante observar que, más allá de las características propias de cada publicación y de su disciplina, no hay recetas secretas ni mágicas para realizar un buen trabajo y llevar a la publicación por la "senda del éxito". ¿Por qué unas sí y otras no? ¿Qué las hace especial? Son preguntas que varios editores se harán al momento de revisar este texto y verificar que el trabajo realizado no difiere del de estas revistas, que por sus características son casi idénticas. Este es el resultado de este tipo de sistemas que, más que certezas, regalan incertidumbre a una comunidad editorial que desde hace décadas conoce muy bien cómo hacer su trabajo con calidad y que de la noche a la mañana es desestimado por "las nuevas reglas", las nuevas políticas de calidad asociadas al "impacto" y a la cita. El análisis inicial es positivo: el hecho de que estas revistas integren esta base de datos es una buena señal para la comunidad disciplinaria en este ámbito en México; al mismo tiempo, es alentador darnos cuenta de que las características generales de estas revistas son las mismas que cumplen otras revistas y que la posibilidad de que puedan ingresar a la base de datos está a un par de clics de distancia. El vacío queda en torno a la elección o a la forma de la integración de estas revistas en relación con otras. Lo negativo es la saturación y delimitación de un sistema editorial que era abierto, libre y reconocido por sus comunidades, que funcionaba sin 
mayores presiones ni pretensiones. Hoy estamos frente a un sistema cerrado que genera presión y pretensión, que se valida desde la propia comunidad y la política pública.

De acuerdo con la información presentada y analizada, podemos ver que a pesar de que estas revistas estén integradas en la base de datos Scopus, sus niveles de citación no son significativos ni tampoco tienen una relación directa con la cantidad de artículos que publica. Al mismo tiempo, no se observa algún indicador administrativo/operativo claro que establezca un parámetro para saber si su estructura y funcionamiento tienen que ver con la permanencia en la base de datos y en el cuartil. La única información que se puede vincular es la cantidad de citas y la posición en el cuartil. Dos de las revistas tuvieron diez o más citas en el periodo evaluado, y coincidentemente en ese periodo escalaron al siguiente cuartil.

Para finalizar, es necesario reflexionar sobre las políticas editoriales que rigen las publicaciones científicas y las instituciones que les dan soporte, ver y analizar si persiguen los mismos objetivos que dieron vida a esa publicación. No todo se reduce a citas y a impacto; el quehacer editorial es mucho más que eso, es mucho más rico y aporta muchos más elementos para el desarrollo de la ciencia y de los científicos.

\section{Referencias bibliográficas}

López-Ornelas, Maricela y Cordero-Arroyo, Graciela, "Un intento por definir las características generales de las revistas académicas electrónicas", Revista Razón y Palabra, 2005, vol. 10, núm. 43, pp. 1-33. Disponible en: http://eprints.rclis.org/15700/

Nassi-Calò, Lilian, "La miopía de los indicadores bibliométricos" [online]. SciELO en Perspectiva, 2017. Disponible en: https://blog.scielo.org/es/2017/06/01/lamiopia-de-los-indicadores-bibliometricos/

\section{Sistemas de información consultados}

Matriz de Información para el Análisis de Revistas. Disponible en: http://miar.ub.edu/ 
Scopus [página web]. Scopus Preview. Disponible en: https://www.scopus.com/sources

Scimago Journal \& Country Rank [página web]. Journal Rankings. Disponible en: https://www.scimagojr.com/journalrank.php

Revista Boletín Mexicano de Derecho Comparado [portal web]. Disponible en: https://revistas.juridicas.unam.mx/index.php/derecho-comparado

Cuestiones Constitucionales. Revista Mexicana de Derecho Constitucional [portal web]. Disponible en: https://revistas.juridicas.unam.mx/index.php/cuestionesconstitucionales

Revista Anuario Mexicano de Derecho Internacional [portal web]. Disponible en: https://revistas.juridicas.unam.mx/index.php/derecho-internacional

Norteamérica. Revista Académica del CISAN-UNAM [portal web]. Disponible en: http://www.revistanorteamerica.unam.mx/index.php/nam

Revista Problema. Anuario de Filosofía y Teoría del Derecho [portal web].

Disponible en: https://revistas.juridicas.unam.mx/index.php/filosofia-derecho

Revista Latinoamericana de Derecho Social [portal web]. Disponible en: https://revistas.juridicas.unam.mx/index.php/derecho-social

SciELo México [portal web]. Disponible en: http://www.scielo.org.mx/scielo.php

Conricyt. Sistema de Clasificación de Revistas Mexicanas de Ciencia y Tecnología (CRMCYT). Disponible en: http://www.revistascytconacyt.mx/index.php/inicio

Dialnet. Fundación Dialnet. Universidad de la Rioja. Disponible en: https://dialnet.unirioja.es/

Sistema Regional de Información en Línea para Revistas Científicas de América Latina, el Caribe, España y Portugal (Latindex). Catálogo 2.0. Disponible en: https://www.latindex.org/latindex/inicio

Red de Revistas Científicas de América Latina y el Caribe, España y Portugal (Redalyc). Universidad Autónoma del Estado de México. Disponible en: https://www.redalyc.org/home.oa

Red Iberoamericana de Innovación y Conocimiento Científico (Redib). Consejo Superior de Investigaciones Científicas (España). Disponible en: https://www.redib.org/?!ng=es 
Master Journal List. Web o Science Group. Clarivate Analytics. Disponible en: https://mjl.clarivate.com/home

Directory of Open Access Journals (DOAJ). Disponible en: https://doaj.org/ 